\title{
Scanning of irradiated silicon detectors using alpha particles and low energy protons
}

\author{
G. Casse ${ }^{\mathrm{a}}$, Z. Doležal $^{\mathrm{b}}$, M. Glaser ${ }^{\mathrm{a}}$, Z. Kohout ${ }^{\mathrm{c}}$, J. Koníček $^{\mathrm{d}}$, \\ F. Lemeilleur ${ }^{\mathrm{a}}$, C. Leroy ${ }^{\mathrm{e}}, \mathrm{V}$. Linhart ${ }^{\mathrm{d}}$, J.J. Mareš ${ }^{\mathrm{f}}$, \\ S. Pospíšil ${ }^{\mathrm{d}, 1}, \mathrm{P}_{\text {. Roy }}{ }^{\mathrm{e}}, \mathrm{B}$. Sopko ${ }^{\mathrm{c}}, \mathrm{M}$. Šiňor ${ }^{\mathrm{d}}$, J. Švejda $^{\mathrm{b}}$, \\ V. Vorobel $^{\mathrm{b}}$, I. Wilhelm ${ }^{\mathrm{b}}$

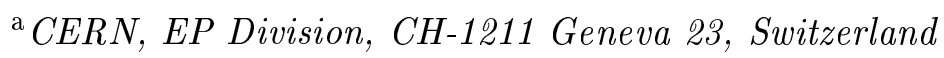 \\ ${ }^{\mathrm{b}}$ Nuclear Center, Faculty of Mathematics and Physics, Charles University, \\ $V$ Holešovičkách 2, Praha 8, CZ-180 00, Czech Republic. \\ ${ }^{\mathrm{c}}$ Department of Physics, Faculty of Mechanical Engineering, Czech Technical \\ University, Technická 4, CZ-166 07 Praha 6, Czech Republic \\ ${ }^{\mathrm{d}}$ Department of Physics, Faculty of Nuclear Sciences and Physical Engineering, \\ Czech Technical University, Břehová 7, Praha 1, CZ-115 19, Czech Republic. \\ ${ }^{\mathrm{e}}$ Department of Physics, University of Montreal, Montreal, H3C 3J7, Canada \\ ${ }^{\mathrm{f}}$ Institute of Physics, Academy of Sciences of the Czech Republic, Cukrovarnická \\ 10, CZ-162 53 Praha 6, Czech Republic
}

RD48/ROSE collaboration

\begin{abstract}
In a spectroscopic study of non-irradiated and proton-irradiated silicon diodes, the detectors were illuminated from the front side and from the rear side by various alpha particle sources (mainly ThC') and by monoenergetic protons with energies from 1.0 to $2.5 \mathrm{MeV}$. Their response characteristics have been studied as a function of the incoming particle energy and the applied bias voltage. The charge collection efficiency was determined as a function of fluence.
\end{abstract}

$\overline{1}$ Corresponding author. E-mail Pospis@mbox.cesnet.cz 


\section{Introduction}

Precise spectroscopy is a powerful tool for investigating semiconductor detector properties. Pulse-height spectra are obtained by measuring the signal of silicon diodes with a standard spectroscopy system. Pulse-height spectra are recorded at various applied bias voltages for silicon diodes whose sensitive volume is scanned with heavy charged particles (alpha's and protons) of low energy and well defined range. The variation of the peak position with the incoming particle energy and with the applied bias provide rather precise information on spatial efficiency of charge collection controlled mainly by the electric field configuration inside the semiconductor. The charge collection efficiency $(\mathrm{CCE})$ is determined from the pulse-height spectra as a function of irradiating proton fluence $(\Phi)$ and applied bias voltage.

\section{Experimental}

Pad diodes of $5 \times 5 \mathrm{~mm}^{2}$ area used for this study were fabricated by SINTEF [1] with planar technology. The junction was formed by implanting $60 \mathrm{keV}$ boron ions to a depth of $0.3 \mu \mathrm{m}$. The front side structure of the diode consists of a $\mathrm{p}^{+}$layer with sheet resistance $R^{\square}=23 \Omega$ covered by an aluminium electrode, leaving a $2 \mathrm{~mm}$ diameter round window in the centre of the detector (for laser illumination). There is an $\mathrm{n}^{+}$layer on the back side with an aluminium grid as electrode. The diodes were each equipped with a guard ring, separated from the main junction by $50 \mu \mathrm{m}$, with a $\mathrm{p}^{+}$implantation depth of less than $1 \mu \mathrm{m}$. The ohmic contact with sheet resistance $R^{\square}=39 \Omega$ was formed by implanting phosphorus ions with an energy of $150 \mathrm{keV}$ to a depth of $\approx 0.3 \mu \mathrm{m}$. An aluminium grid of $500 \mu \mathrm{m}$ pitch formed by $50 \mu \mathrm{m}$ wide strips is deposited on this ohmic side. Nine identical n-type silicon diodes with a thickness of $\sim 280 \mu \mathrm{m}$ and initial resistivity of $\sim 2200 \Omega \mathrm{cm}$ were selected. Eight of them were irradiated with $24 \mathrm{GeV} / \mathrm{c}$ protons in the CERN-PS T7 beam.

A multiple alpha source was prepared for the measurements with alpha particles under vacuum [2]. It contained ${ }^{239} \mathrm{Pu},\left({ }^{241} \mathrm{Am}\right), \mathrm{ThC}$, and ThC' which emit alpha particles of energy $5.157 \mathrm{MeV},(5.486 \mathrm{MeV}), 6.051 \mathrm{MeV}$ and $6.090 \mathrm{MeV}$, and $8.784 \mathrm{MeV}$, respectively. The ranges of these $\alpha$-particles in silicon are listed in Table 1. The proton measurements were performed at the Charles University van de Graaff accelerator with energies from 1.0 up to $2.5 \mathrm{MeV}$. The protons from the direct beam were backscattered on a thin gold foil to decrease the flux. The ranges of these protons in silicon are also shown in Table 1.

A standard spectroscopy system composed of a charge-sensitive preamplifier, 
linear shaping amplifier and multichannel analyser was used to record pulseheight spectra obtained from $\alpha$-particle and proton illumination of the front side and back side of the diodes. The measurements were performed by applying a bias voltage to the front electrode and also to the guard ring. A silicon surface barrier detector was used as reference detector. This detector had a thin entrance window, very good energy resolution and $100 \%$ charge collection efficiency. The normalisation of the peak position observed with the various diodes to that observed with the reference detector was used to determine CCE. The full width at half maximum (FWHM) at $5.486 \mathrm{MeV}\left({ }^{241} \mathrm{Am}\right.$ alpha-line) was $14 \mathrm{keV}$, giving a relative energy resolution of $0.26 \%$ for the whole spectroscopy system (reference detector included). This good resolution enabled us to observe changes in pulse height resulting from the changes in charge collection efficiency (CCE) or from the influence of the electrode structures.

The scanning of the diode structure and its sensitive volume by illuminating the front side and the back side with heavy charged particles (alpha's and protons) of low energy and well defined range is shown schematically in Fig. 1. The dependence of the depth of the depleted (sensitive) layer on the applied bias voltage is compared with the Bragg curve distribution of charge produced along the path of the incoming particle.

The sensitivity of the method is illustrated in Fig. 2 where the response of the reference detector (Fig. 2a) is compared with the responses of a nonirradiated detector illuminated from the front side (Fig. 2b) and from the back side (Fig. 2c). From the splitting and shifts of the $\alpha$-peak, one can determine the thicknesses of metallic electrodes and of implanted layers as well. The possibility of measuring shifts at the level of a $\mathrm{keV}$ is equivalent to measuring layer thicknesses of about ten nanometers.

\section{Results}

The diodes exposed to different fluences of $24 \mathrm{GeV} / \mathrm{c}$ protons in the CERNPS T7 beam exhibit different values of full depletion voltage, as shown in Fig. 3. The full depletion voltage was measured with the $\mathrm{C}-\mathrm{V}$ method. These diodes were scanned with alpha-particles and protons for various applied bias voltages.

Some of the spectra illustrating the response of the non-irradiated detector to $\alpha$ particles impinging on the front side of the diode are shown in Fig. 4 for different values of applied reverse bias $(U=0.0,8.7,80 \mathrm{~V})$, with a shaping time $\tau=0.25 \mu \mathrm{s}$. According to Fig. 3, full depletion of the detector is achieved at

$U_{f d} \approx 115 \mathrm{~V}$. The energy resolution of the peaks is maintained over the whole 
voltage range from the lowest to the highest value when the depletion depth is larger than the particle penetration depth. The presence of satellite peaks at low amplitudes depends on the voltage applied on the guard ring. These satellites disappear when the guard ring voltage is switched off. This effect can be qualitatively explained as a signal induced by the charge generated in the volume close to the guard ring, similar to that observed in classical ionisation chambers [3]. The induced signal from the guard ring circuitry produces its own shifted energy spectrum, superimposed on the detector regular spectrum. The complete understanding of this behaviour will need further investigation.

Spectra illustrating the response of the non-irradiated diode to $\alpha$ particles incident on the back side are shown in Fig. 5 as a function of voltage, for the same shaping time $(\tau=0.25 \mu \mathrm{s})$. The signal starts to be observed at higher voltage compared with front side illumination, as expected. However, unexpectably, a sizeable signal can be observed even at a voltage (e.g. $62 \mathrm{~V}$ ) at which the corresponding depleted region is far from reaching the back side of the diode (the undepleted depth corresponding to $62 \mathrm{~V}$ is $80 \mu \mathrm{m}$ ). The pulses are spread into broad peaks. As the voltage is increased, all amplitudes are also increased and the response changes into sharp peaks with left-hand side asymmetry. The size of this asymmetry decreases with increasing voltage and eventually disappears completely. This behaviour could be caused by the back side contact structure influencing the shape of the depleted layer. A response as good as the one obtained for front exposure is achieved when the applied voltage is high enough to deplete the detector fully.

The observed back side signal at relatively low voltage can possibly be explained by assuming the existence of a spatial inhomogeneity of the electric field along the back contact plane. This effect can be understood using a model based on the behaviour of a set of detectors connected in parallel, each biased at a slightly different voltage. The effect of the pulses spread towards lower values has the same origin as the observed shifts of some peaks due to decreased bias voltage applied to the detector, as depicted in Fig. 5. At lower bias and for rear side illumination the ionising track does not reach the sensitive volume of the detector completely. Consequently, the collection of the charge produced is not optimal and the influence of electric field inhomogeneities becomes decisive, in that small local changes in the electric field can effectively diminish or even remove the gap between the depletion layer and the front of the ionising particle track and can therefore switch the corresponding part of the detector structure into a regime where the charge collection efficiency is considerably higher. With increasing bias, all parts of the detector eventually achieve their maximum and equal charge collection efficiency, leading to the disappearence of the asymmetric spread among pulse amplitudes. These electric field inhomogeneities are possibly caused by the special rear contact design of the detectors under study. Prior to the aluminium metallisation, a $0.3 \mu \mathrm{m}$ thick $\mathrm{n}^{+}$layer was implanted on the whole surface of undoped bulk ma- 
terial. While the conductivity of aluminium $\left(\approx 2.7 \times 10^{-8} \Omega \mathrm{m}\right)$ is four orders of magnitude higher than that of the implanted layer $\left(\approx 1.2 \times 10^{-4} \Omega \mathrm{m}\right)$, the voltage drops in the metallic grid can be neglected and the problem reduces to the investigation of the potential distribution along the interface between the $\mathrm{n}^{+}$layer and the bulk material.

Via lateral transport, the implanted $\mathrm{n}^{+}$layer with sheet resistance $R^{\square}=39 \Omega$ equalizes the potentials at the interface between the $\mathrm{n}^{+}$layer and the bulk material. However, this equalization is not perfect. The change of voltage ratio $U / U_{0}$ with distance $x$ from the metallised edges can be approximated using the transmission line formula [4]

$$
\frac{U}{U_{0}}=\exp \left(-x \sqrt{R^{\square} / R_{s}}\right)
$$

where $R_{s}$ is the specific interface resistance between the $\mathrm{n}^{+}$layer and the bulk material, measured in units of $\Omega \mathrm{m}^{2}$. This quantity is mainly controlled by the properties of the accumulation layer [4]. In the present case the mobility and the accumulation layer thickness (i.e. diffusion length) are $\mu=0.145 \mathrm{~m}^{2} / \mathrm{Vs}$ and $\lambda=5 \mu \mathrm{m}$ (cfr. [5]), respectively. Then $R_{s}$ is given by:

$$
R_{s}=\lambda / e \mu n_{j}
$$

where $n_{j}$ is the excess charge concentration in the accumulation layer. The determination of $n_{j}$, using statistics, is a rather questionable task because of the strong degeneracy of the electron gas involved [4]. Nevertheless, the difficulty can be overcome by taking into account that the transport through the structure should be continuous. The comparison between the current density $\sim e n_{i} v\left(n_{i}\right.$ is the intrinsic electron concentration and $v$ the thermal velocity, for Si: $n_{i} \approx 1 \times 10^{16} \mathrm{~m}^{-3}$ and $v \approx 5 \times 10^{4} \mathrm{~m} / \mathrm{s}$ ) and the current density $\sim e n_{j} \lambda / \epsilon \epsilon_{0}$ due to the local electric field $\left(\epsilon \epsilon_{0}\right.$ is the permittivity, $\epsilon=12$ for $\mathrm{Si})$ in the accumulation layer leads to a continuity equation of the form [5]:

$$
n_{j}{ }^{2}=n_{i} v \epsilon \epsilon_{0} / e \mu \lambda
$$

This formula provides the values: $n_{j}=6.8 \times 10^{17} \mathrm{~m}^{-3}$ and $R_{s}=3.2 \times 10^{-4} \Omega \mathrm{m}^{2}$. Substitution of the latter value into eq. (1) gives the result that the difference between voltages at the interface point $x=0$ (metallisation edge) and at $x=200 \mu \mathrm{m}$ (centre of the square grid) is about $7 \%$ of the total applied voltage $U_{0}$. Therefore, this simple model gives an estimation of differences between voltages on the rear contact plane which are not negligible when compared to the applied voltage itself. Such differences could explain the observation of broadened peaks with left-hand side asymmetry at the rear side illumination when the detector is close to full depletion. 
Examples of spectra illustrating the response of an irradiated diode $(\Phi=$ $\left.5.2 \times 10^{13} \mathrm{p} / \mathrm{cm}^{2}\right)$ to illumination by $\alpha$ particles on both sides of the diode as a function of voltage and for the shaping time $\tau=0.25 \mu$ s are shown in Fig. 6 (front side) and Fig. 7 (back side). For the front exposure, the peak resolution is degraded as a consequence of decreased charge collection efficiency. With increased bias, the amplitude increases and the resolution improves but without achieving the resolution observed before irradiation, due to increased reverse current. Qualitatively the collected charge and resolution are determined by the collection time and, therefore, by charge carrier lifetime and electric field. As seen from Fig. 3, this irradiated detector is close of being made of semiinsulating material. This view is supported by the results obtained from the back side illumination. The trend is similar to that observed for the front side spectra.

The evolution of the spectroscopic response of the detector as a function of fluence is shown in Fig. 8 and 9 for protons and $\alpha$ particles, respectively. The dependence of the peak position, which corresponds to the collected charge, on incoming particle energies (protons in Fig. 8 and alpha particles in Fig. 9) is shown as a function of the applied voltage for front side and back side illumination. At zero or low fluences, the signals for the front side illumination start to be observed at very low voltage, in contrast to the back side signals which are observed for relatively high values of the bias, when the depleted layer is closer to the back side than the particle range. When approaching the inversion point, the front and back signal start to show the same behaviour with the applied voltage (see Fig. 8e and Fig. 9c). Beyond the inversion point, one can see clearly reversed behaviours despite the fact that the spectroscopic measurements were made more complicated by the increased reverse current.

The charge collection efficiency was determined from saturated values of peak positions measured at different voltages compared to the reference detector. The charge collection efficiency as a function of fluence for $8.78 \mathrm{MeV}$ alpha particles (from ThC') and 1.7 MeV protons is shown in Fig. 10. For $8.78 \mathrm{MeV}$ alpha particles incident on the front side of the diodes operated at $144 \mathrm{~V}$ reverse bias, a charge collection deficit of about $18 \%$ is observed at fluence $\Phi \approx 1.7 \times 10^{14} \mathrm{p} / \mathrm{cm}^{2}$, while the charge collection deficit is measured of about $16 \%$ at this fluence for back side illumination. Similarly, a charge collection deficit of about $18 \%$ for $1.7 \mathrm{MeV}$ proton incident on the front and back side of the diode operated at $100 \mathrm{~V}$ bias is measured at $\Phi \approx 1.0 \times 10^{14} \mathrm{p} / \mathrm{cm}^{2}$.

\section{Discussion and Conclusions}

The spectroscopy method has been applied to the study of non-irradiated and proton-irradiated silicon diodes. The pulse-height spectra show the de- 
pendence of the peak position on the incoming particle energy and on the applied bias. A significant influence of the contact structure on the detector has been observed. The observed shift of some peaks to lower channels, as well as their spread and asymmetry, can possibly be explained in terms of a spatial inhomogeneity of the electric field along the back side contact plane. As a consequence, all possible sources of inhomogeneities in the electric field, such as grid structures for the back side contact, should be avoided to ensure uniform response across the detector surface. Conducting-type inversion of the silicon material has been clearly demonstrated from the behaviour of the peak position. Indeed, for zero or low fluences, it has been observed that signals produced by front side illumination are already observed at very low bias, in contrast to the back side signals which are seen for relatively high values of the bias. Around the n-type to p-type inversion, front and back signals show the same behaviour with the applied voltage. Beyond the inversion point reversed behaviours are observed.

The charge collection efficiency (CCE) was measured as a function of fluence $(\Phi)$ and applied bias voltage. Since the number of radiation induced defects increases with the fluence, the charge carrier lifetimes due to trapping decrease and an expected charge collection deficit is observed. After a proton fluence of $2 \times 10^{14} \mathrm{p} / \mathrm{cm}^{2}$, however, the CCE still remains higher than $80 \%$ of the initial value.

\section{Acknowledgements}

This work was supported in part by the Czech Committee for Collaboration with CERN under a Grant of Ministry of Industry and Trade, by the Grant Agency of Academy of Sciences of the Czech Republic under contract GAAVA1010806 and by the Grant Agency of the Czech Republic under contract GAČR-202/99/0410. C.L. and P.R. thank NSERC of Canada for their support.

\section{References}

[1] L. Evensen, Processing of the CERN 2 devices at SINTEF, 2nd Workshop on Radiation Hardening of Silicon Detectors, CERN 4-5 February 1997, Rose Collaboration, CERN RD-48 (1997) 47.

[2] Č. Jech, Z. Janout, J. Koníček, J. Kubašta, S. Pospíšil and M. Vobecký, Decay characteristics of electrostatically collected samples of radon daughters, Collect. Czech. Chem. Comm. (Vol 62) (1997) 218. 
[3] D.H. Wilkinson, Ionization chambers and counters, Cambridge at the University Press, Cambridge, 1950, pp. 122-123.

[4] H.K. Henisch, Semiconductor contacts, Clarendon Press, Oxford, 1984, pp. 293323.

[5] S.M. Sze, Physics of Semiconductor Devices, John Wiley \& Sons, New York, Second Edition, 1981, pp. 637. 
Table 1

Ranges $(R)$ of protons of energy $T_{p}$ and $\alpha$-particles of energy $T_{\alpha}$ in silicon.

\begin{tabular}{ccccc}
\hline$T_{p}[\mathrm{MeV}]$ & $R\left(T_{p}\right)[\mu m]$ & & $T_{\alpha}[\mathrm{MeV}]$ & $R\left(T_{\alpha}\right)[\mu m]$ \\
\cline { 5 - 5 } 0.5 & 6.64 & & 5.157 & 25.95 \\
1.0 & 16.97 & & 5.486 & 28.33 \\
1.5 & 30.94 & & 5.805 & 32.66 \\
2.0 & 48.30 & & 6.090 & 32.97 \\
2.5 & 68.91 & & 8.784 & 57.38 \\
\hline
\end{tabular}




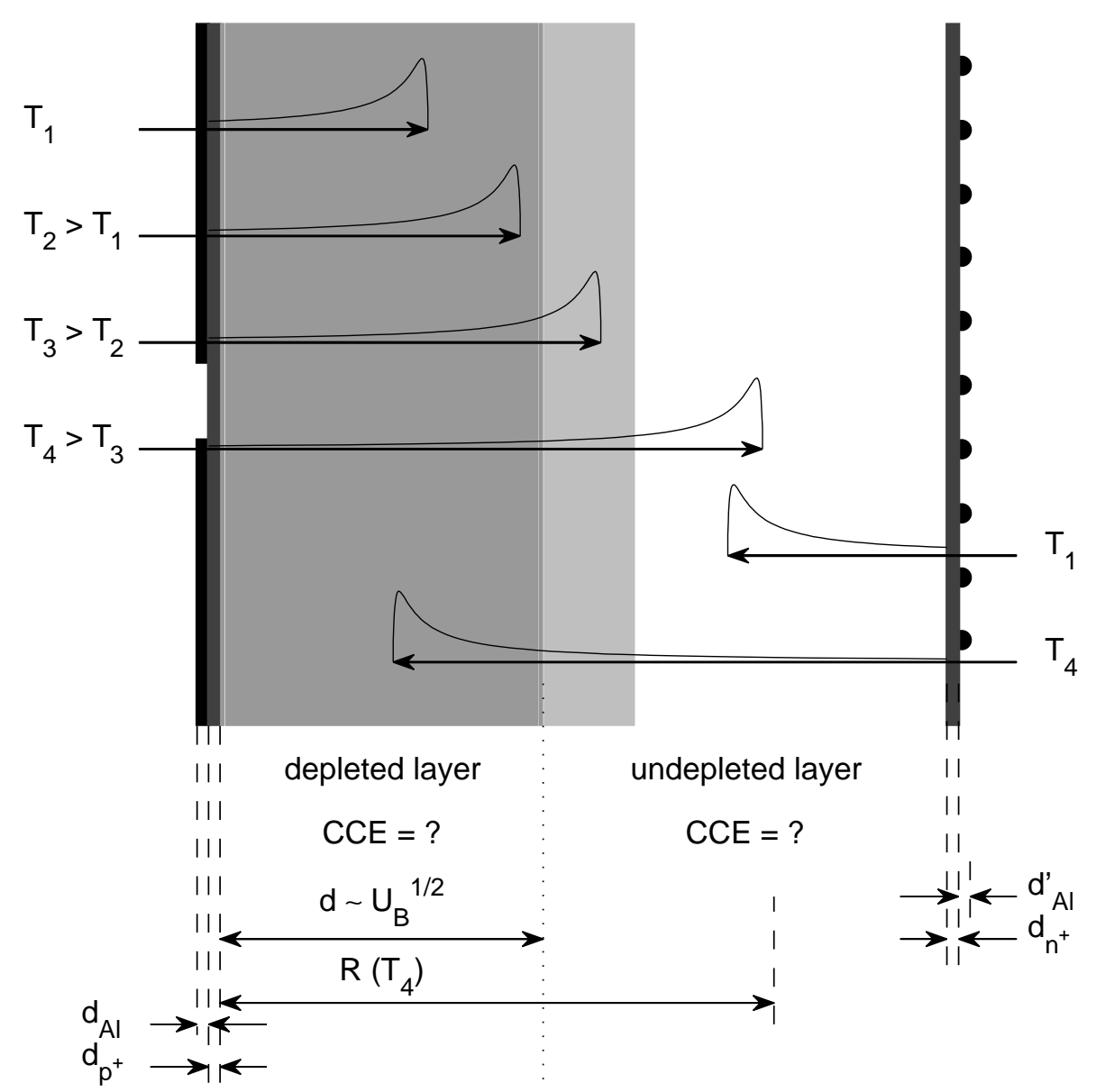

Fig. 1. The method for scanning the detector structure and sensitive volume is illustrated in the schematic cross-sectional view of a detector of thickness $d$. The thicknesses of $\mathrm{Al}$ contact metallisation and $\mathrm{p}^{+}$layer (front side) or $\mathrm{n}^{+}$layer (back side) are indicated. The shaded area represents the depleted region (detector sensitive volume). The detector response is measured as a function of the applied bias voltage for protons or alpha particles incident on the front or on the back side at energies $T_{4}>T_{3}>T_{2}>T_{1}$, corresponding to incoming particle ranges $R\left(T_{4}\right)>R\left(T_{3}\right)>R\left(T_{2}\right)>R\left(T_{1}\right)$ inside the detector. Bragg energy loss distributions inside the detectors are shown for the various incident energies. 


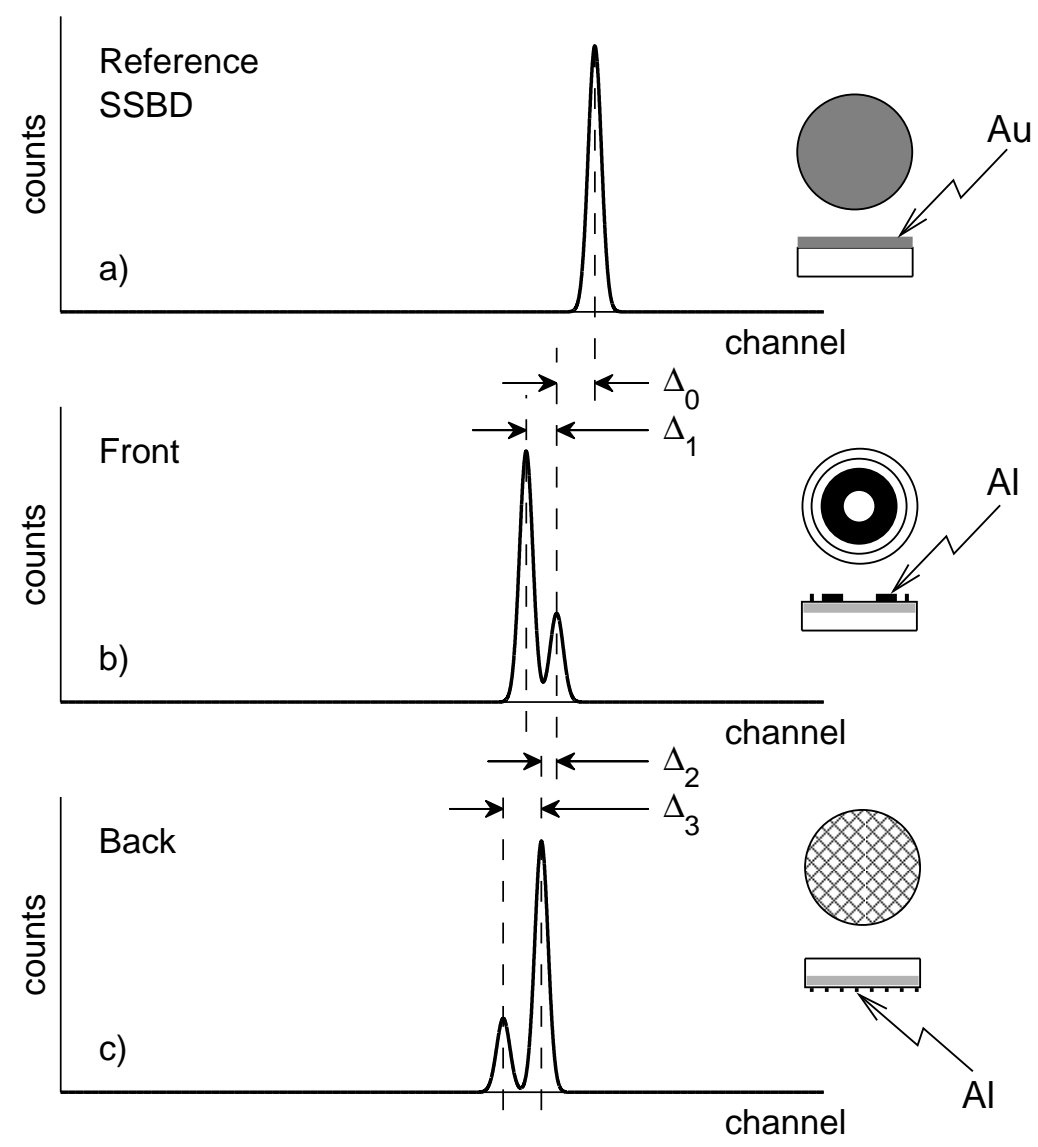

Fig. 2. Illustration of the sensitivity of the spectroscopic method. The response of a reference silicon surface barrier detector (SSBD) a) is compared to the response of a non-irradiated detector illuminated b) on the front side and c) on the back side. Shifts among peaks and their splitting are caused by different losses of particles entering through non-sensitive layers on the detector surface. $\Delta_{0}$ : difference due to different losses in $\mathrm{Au}$ reference detector electrode and $\mathrm{p}^{+}$layer of the measured detector $(\simeq 20 \mathrm{keV}), \Delta_{1}$ : thickness of front $\mathrm{Al}$ electrode $(\simeq 86 \mathrm{keV}), \Delta_{2}$ : backside $\mathrm{n}^{+}$layer thickness compared to $\mathrm{p}^{+}(\simeq 33 \mathrm{keV}), \Delta_{3}$ : backside Al grid thickness $(\simeq 122 \mathrm{keV})$. The thicknesses are expressed in terms of the corresponding energy losses. 


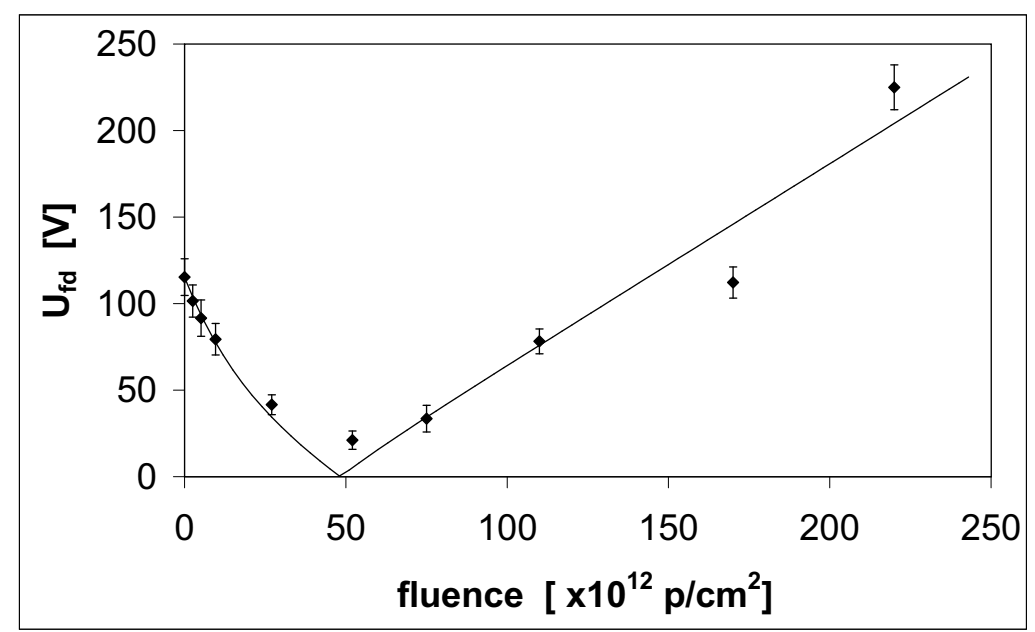

Fig. 3. Full depletion voltage as a function of the accumulated fluence (proton $/ \mathrm{cm}^{2}$ ) for the diodes under study. The full depletion voltage was measured with the $\mathrm{C}-\mathrm{V}$ method. 


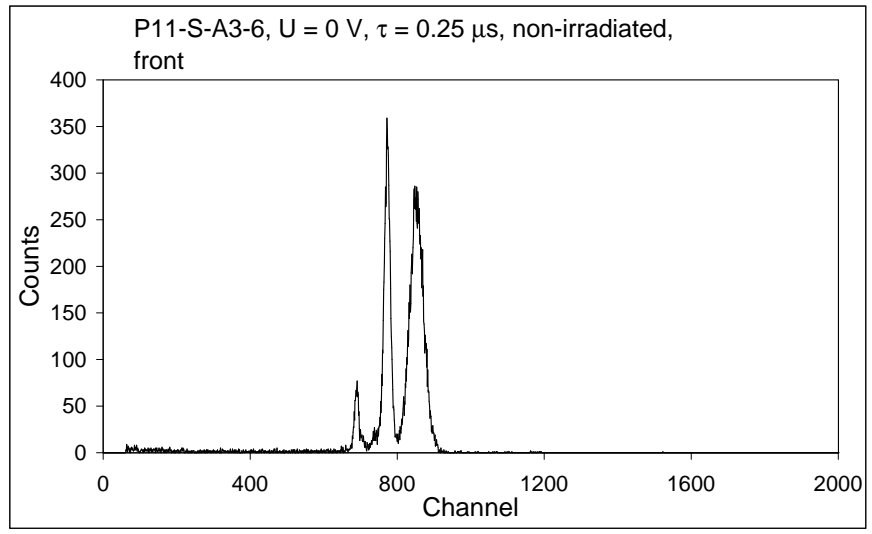

a)

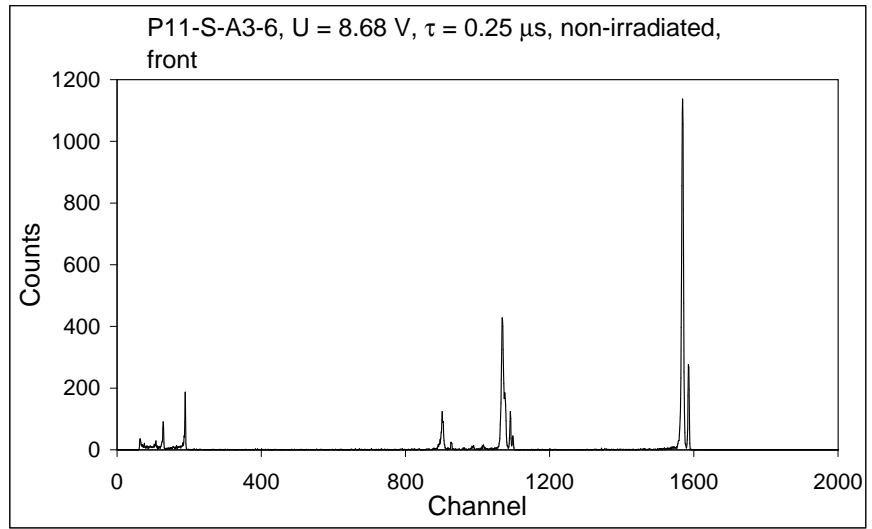

b)

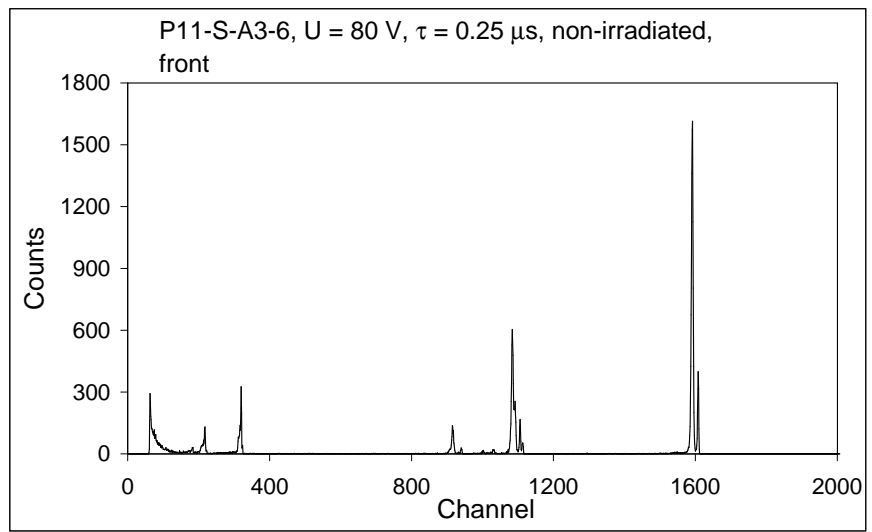

c)

Fig. 4. Spectra illustrating the response of the non-irradiated detector to alpha particles from $\mathrm{Pu}$, ThC and ThC' incident on the front side of the diode for applied reverse bias: $U=0.0$ (a), 8.7 (b) and $80 \mathrm{~V}$ (c). The shaping time was $\tau=0.25 \mu \mathrm{s}$. 


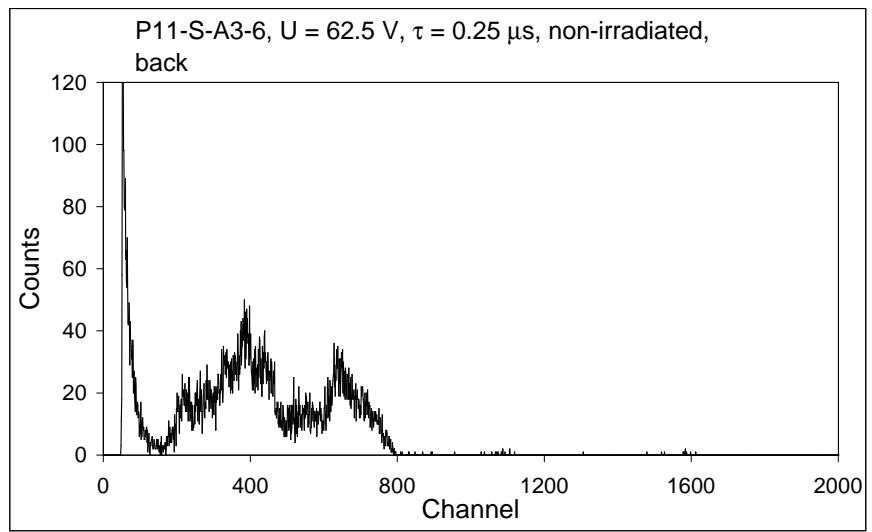

a)

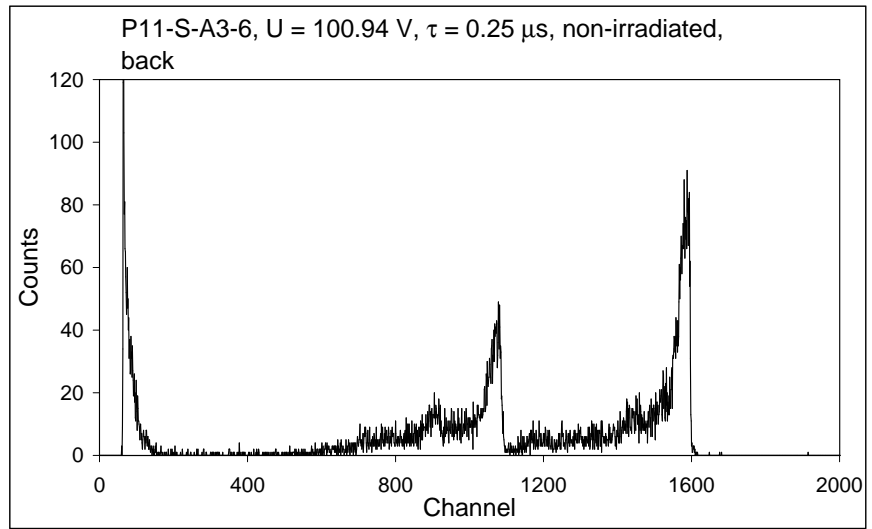

b)

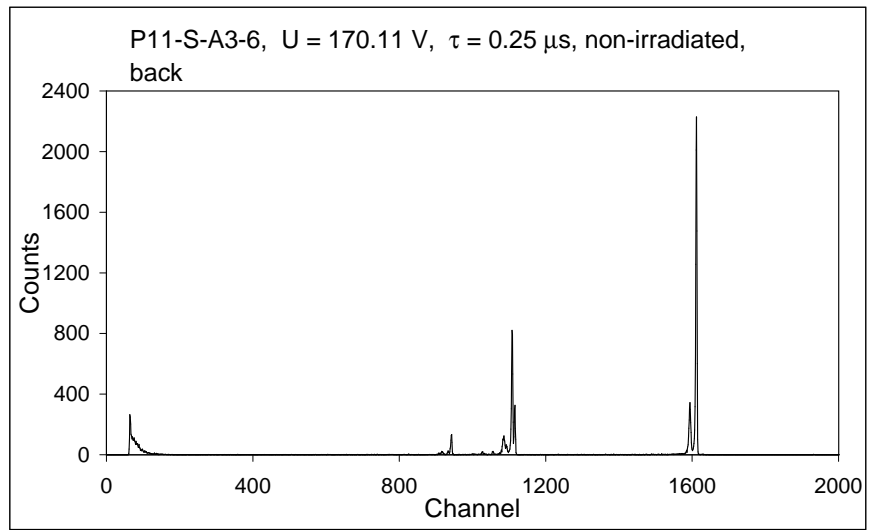

c)

Fig. 5. Spectra illustrating the response of the non-irradiated detector to alpha particles from $\mathrm{Pu}$, ThC and ThC' incident on the back side of the diode for applied reverse bias: $U=62.5$ (a), 100.9 (b) and $170.1 \mathrm{~V}$ (c). The shaping time was $\tau=0.25 \mu \mathrm{s}$. 


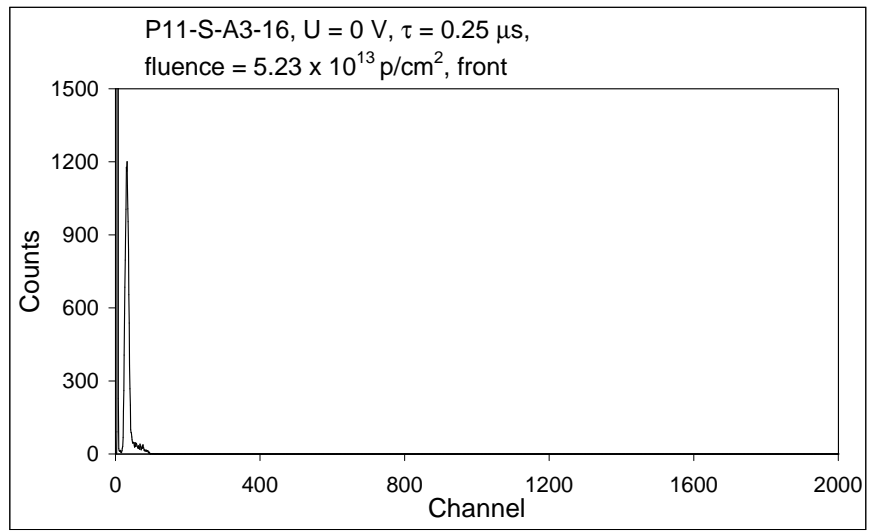

a)

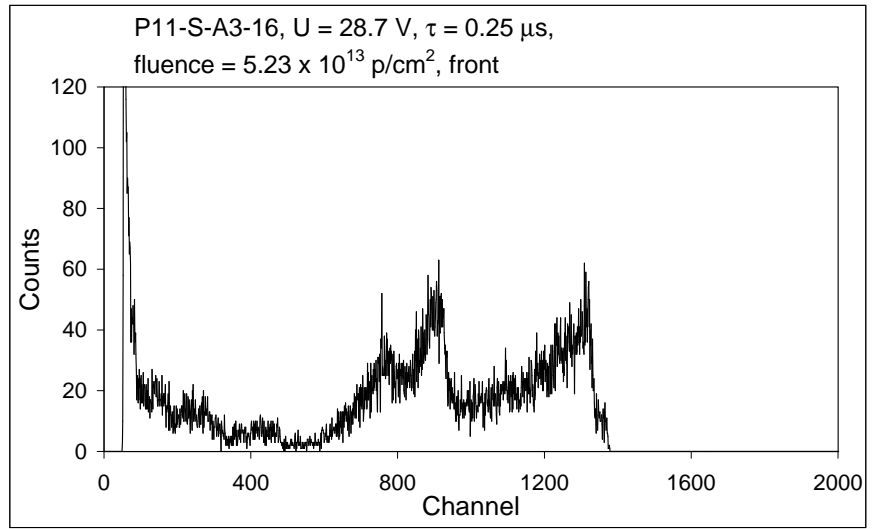

b)

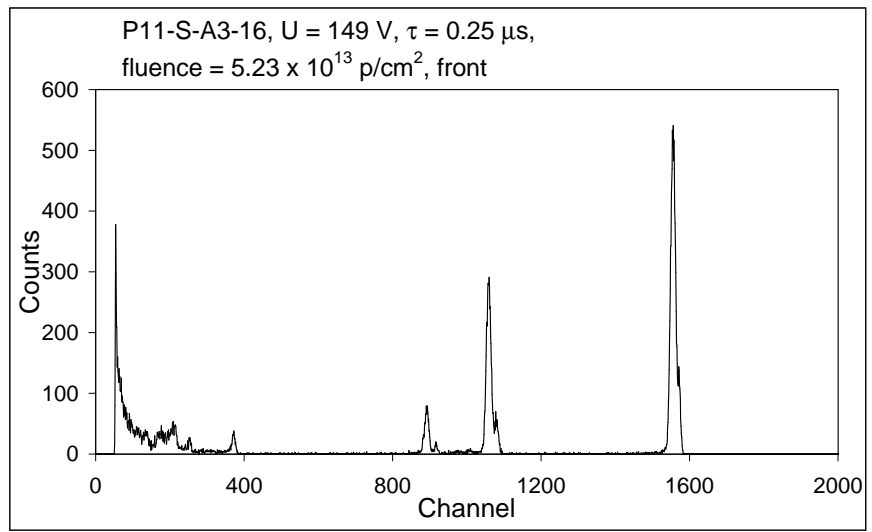

c)

Fig. 6. Spectra illustrating the response of an irradiated detector $\left(\Phi=5.2 \times 10^{13} \mathrm{p} / \mathrm{cm}^{2}\right)$ to alpha particles from $\mathrm{Pu}$, ThC and $\mathrm{ThC}$ ' incident on the front side of the diode for applied reverse bias: $U=0.0$ (a), 28.7 (b) and $149 \mathrm{~V}$ (c). The shaping time was $\tau=0.25 \mu \mathrm{s}$. 


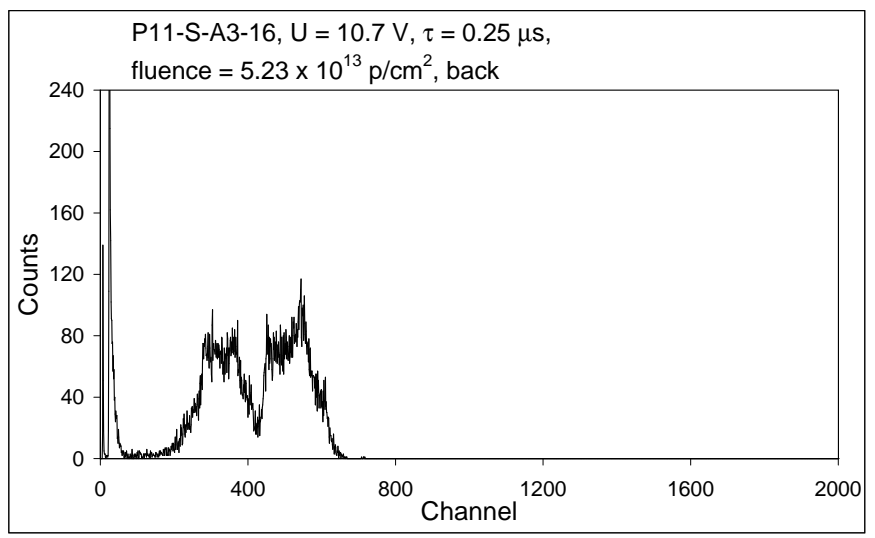

a)

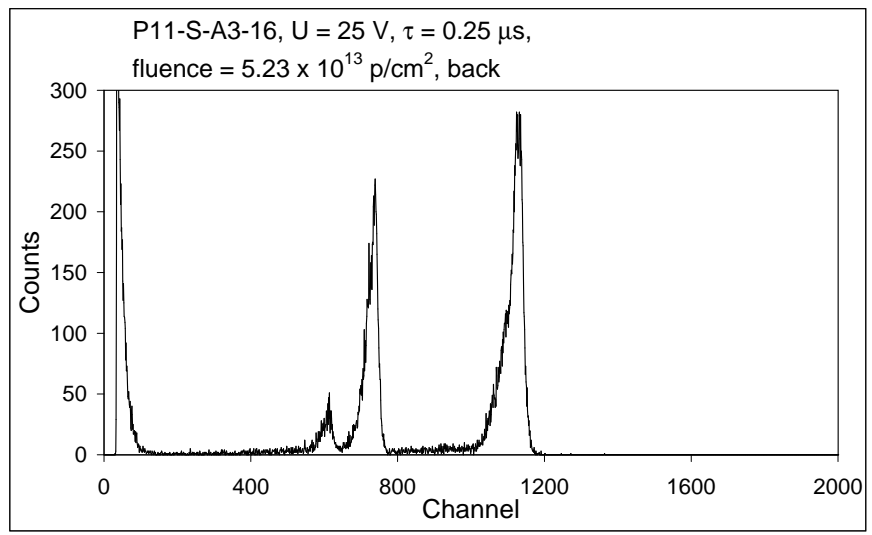

b)

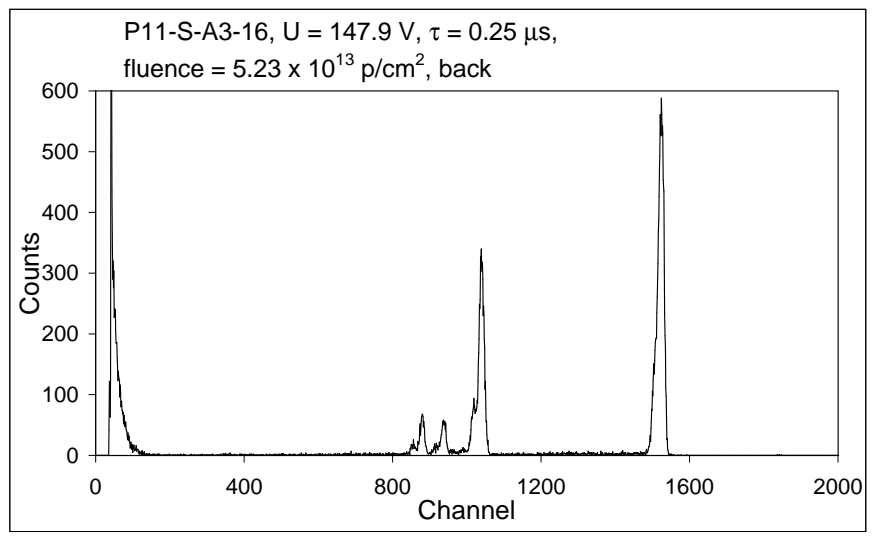

c)

Fig. 7. Spectra illustrating the response of an irradiated detector $\left(\Phi=5.2 \times 10^{13} \mathrm{p} / \mathrm{cm}^{2}\right)$ to alpha particles from $\mathrm{Pu}$, ThC and $\mathrm{ThC}^{\prime}$ incident on the back side of the diode for applied reverse bias: $U=10.7$ (a), 25 (b) and $147.9 \mathrm{~V}$ (c). At bias of $147.9 \mathrm{~V},{ }^{241} \mathrm{Am}$ alpha source was added. The shaping time was $\tau=0.25 \mu \mathrm{s}$. 


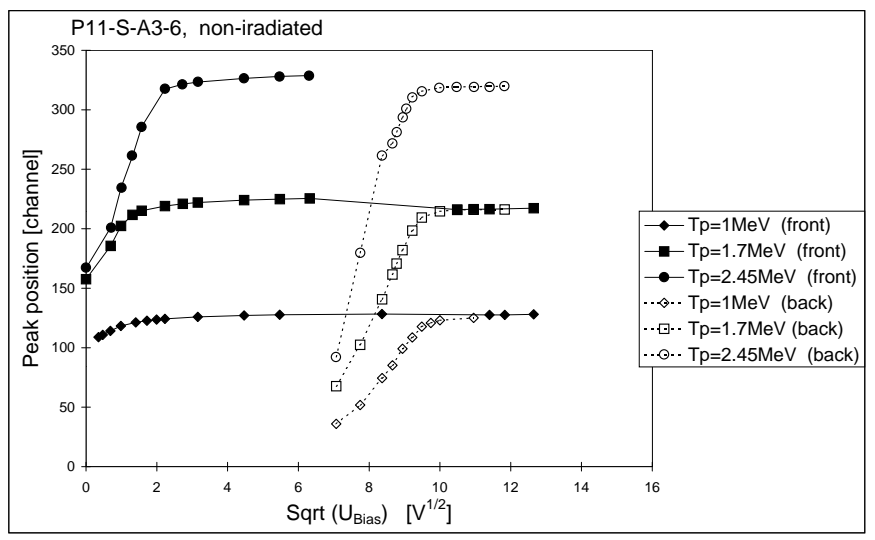

a)

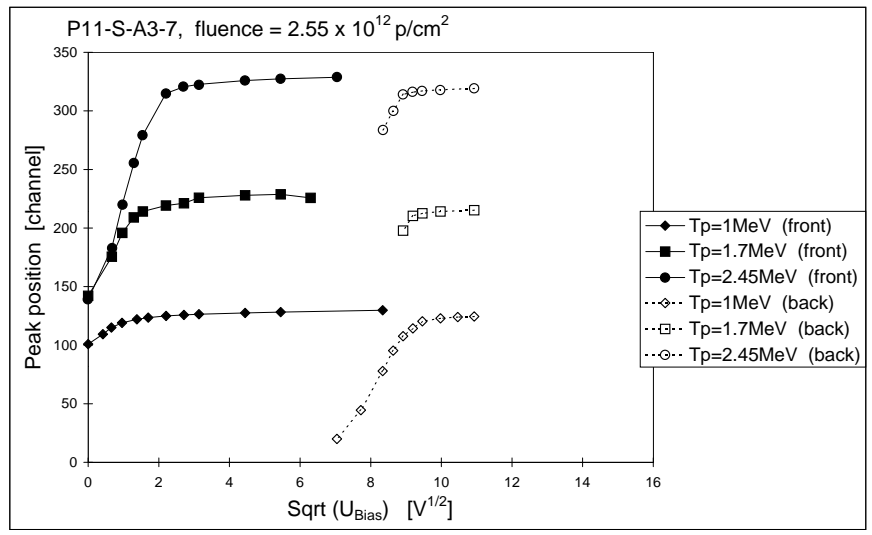

b)

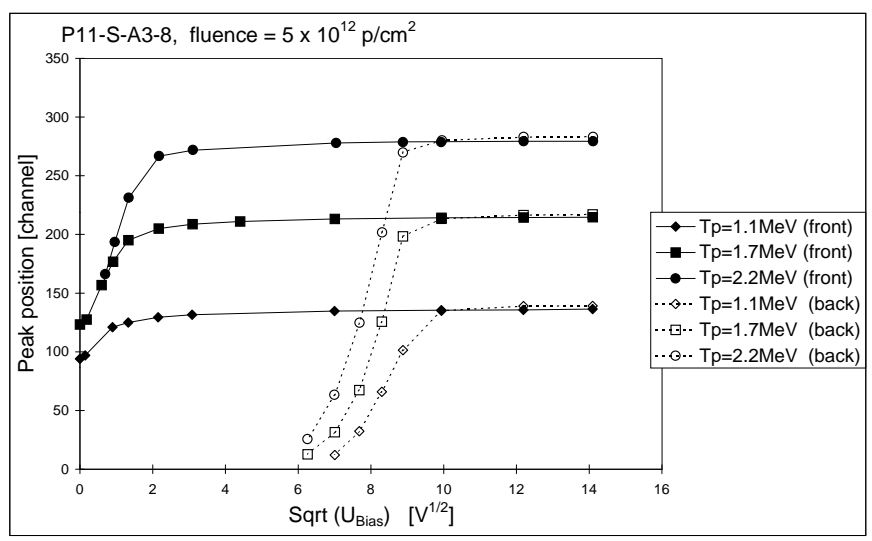

c)

Fig. 8. (a-c) Peak position (expressing the collected charge) dependence on fluence, applied bias voltage, and incoming proton energy for particles incident on the front side and back side of the diode. 


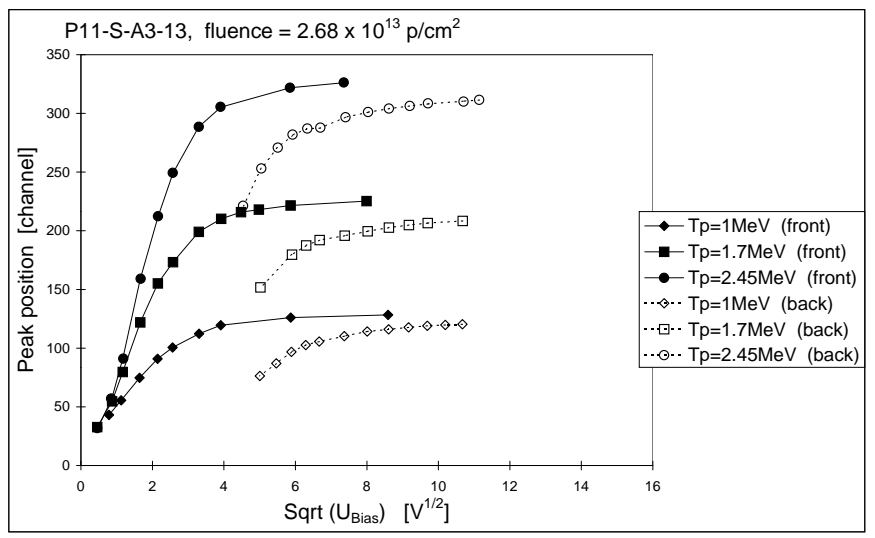

d)

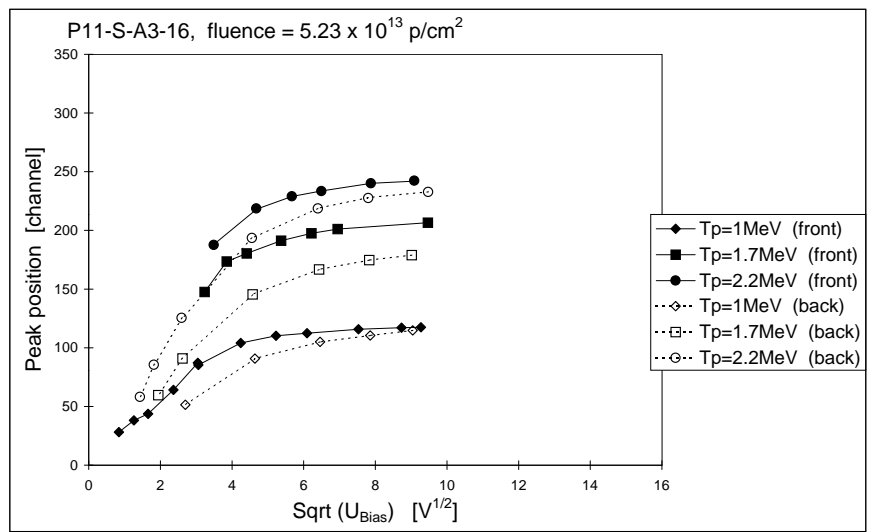

e)

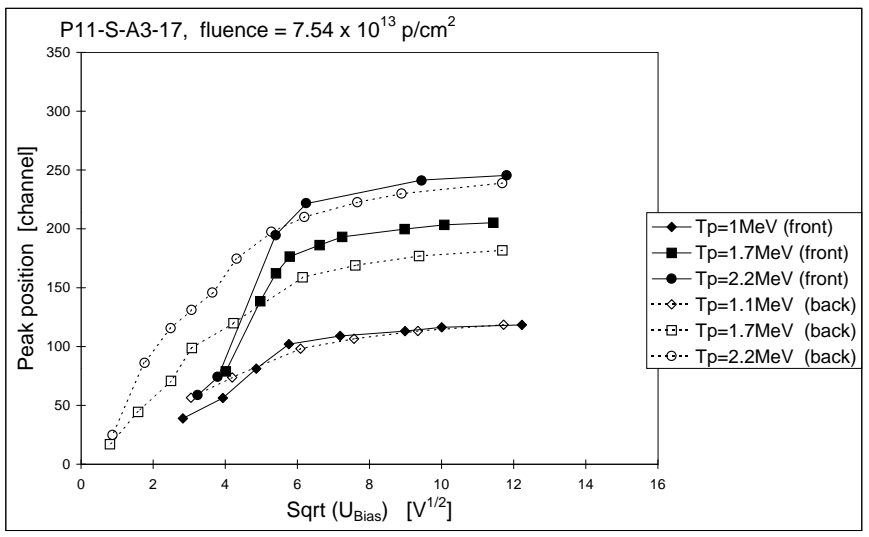

f)

Fig. 8. (d-f) Peak position (expressing the collected charge) dependence on fluence, applied bias voltage, and incoming proton energy for particles incident on the front side and back side of the diode. 


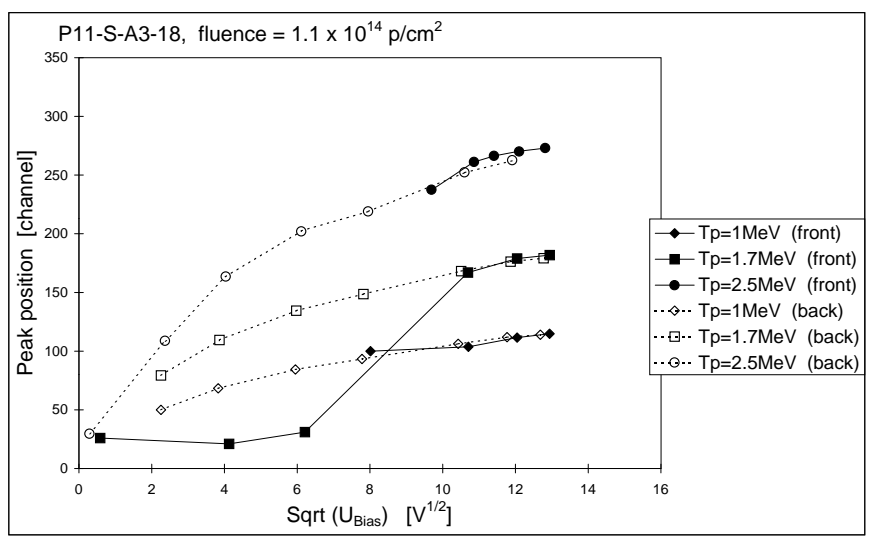

g)

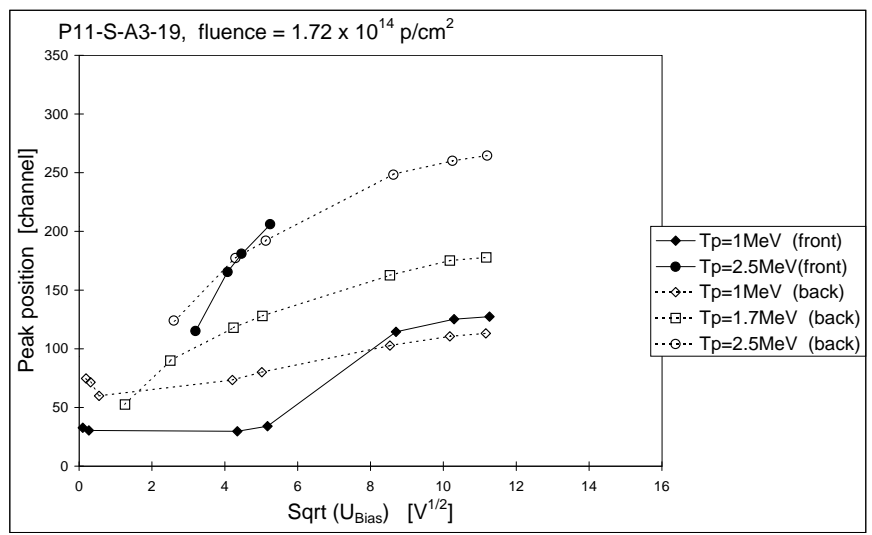

h)

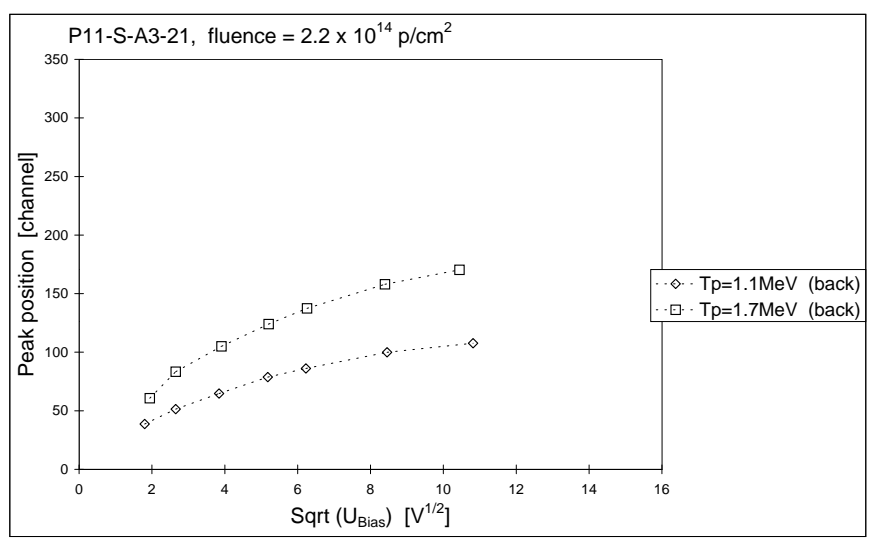

i)

Fig. 8. (g-i) Peak position (expressing the collected charge) dependence on fluence, applied bias voltage, and incoming proton energy for particles incident on the front side and back side of the diode. 


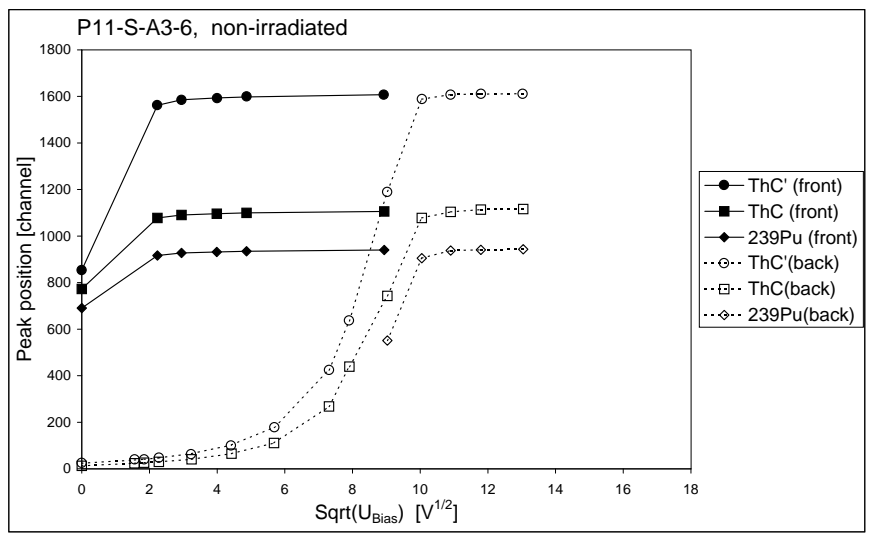

a)

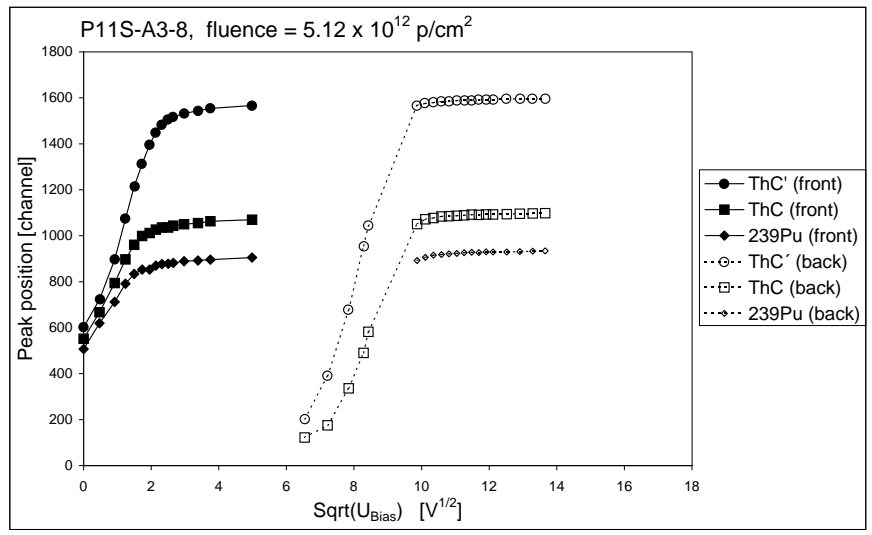

b)

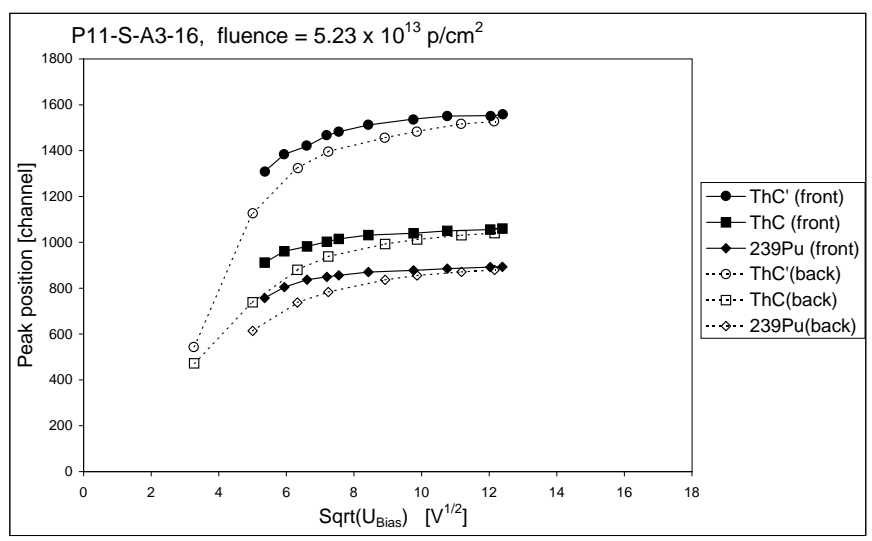

c)

Fig. 9. (a-c) Peak position dependence on fluence, applied bias voltage, and incoming alpha particle energy for particles incident on the front side and back side of the diode. 


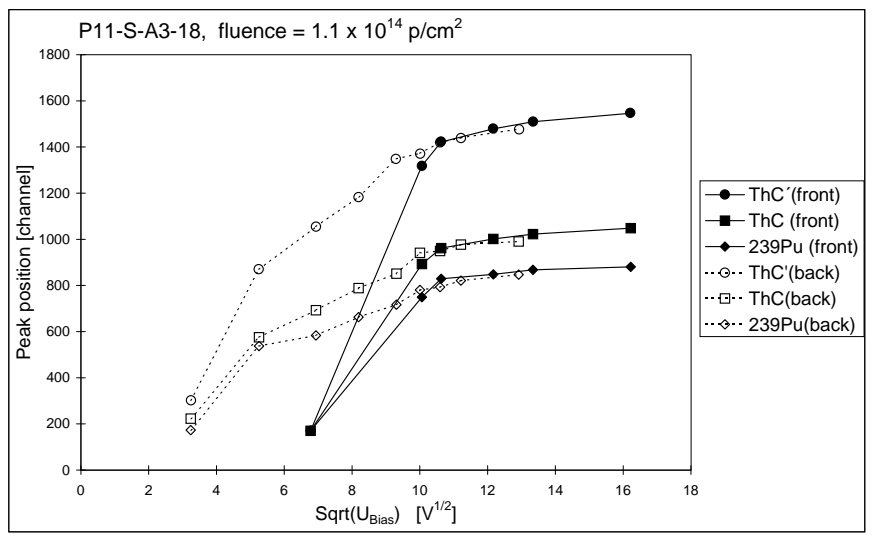

d)

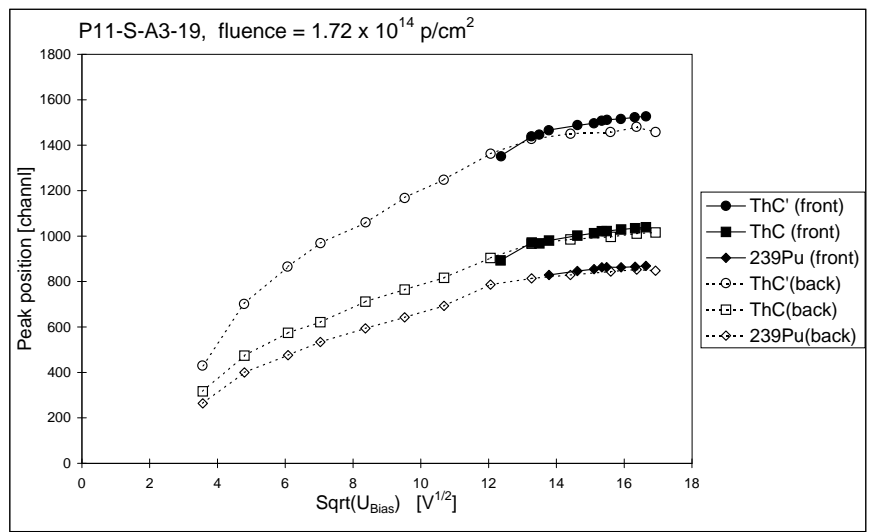

e)

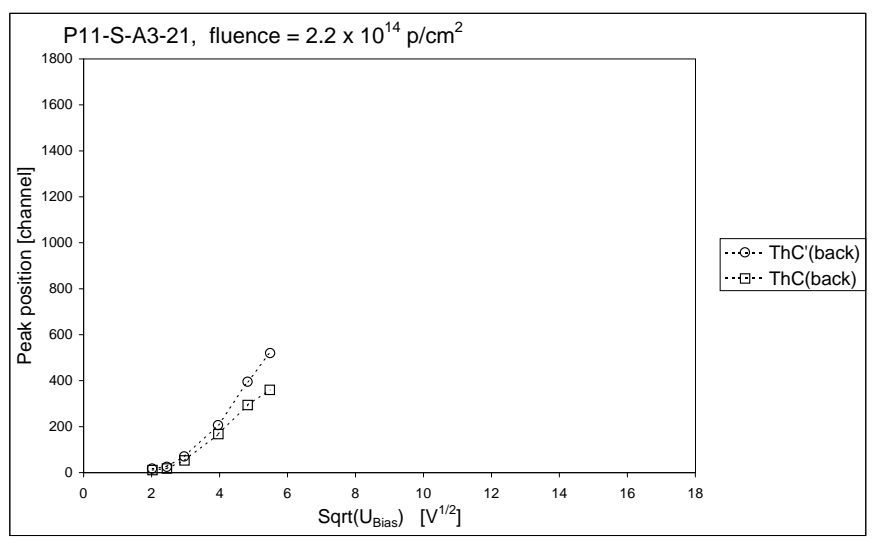

f)

Fig. 9. (d-f) Peak position dependence on fluence, applied bias voltage, and incoming alpha particle energy for particles incident on the front side and back side of the diode. 


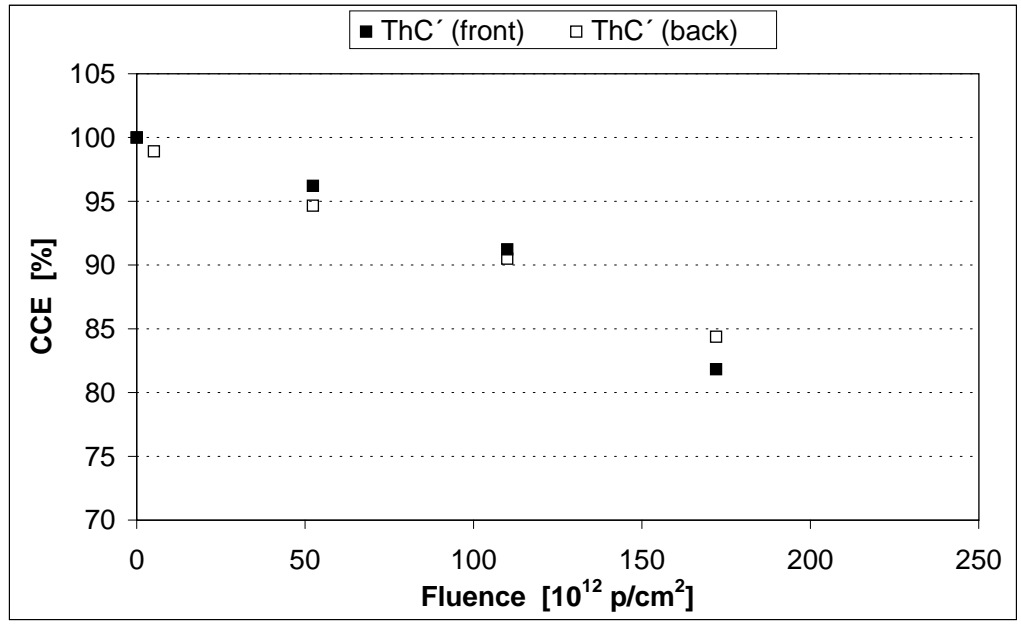

a)

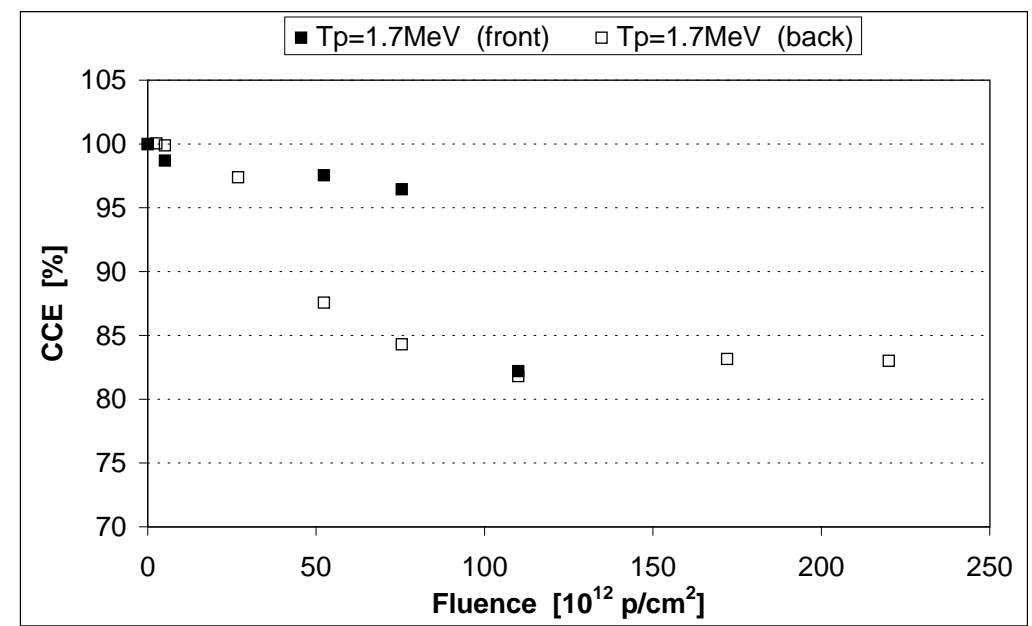

b)

Fig. 10. Charge collection efficiency (CCE) for front and back side illumination of the diode determined by $8.78 \mathrm{MeV}$ alpha particles at $144 \mathrm{~V}$ reverse bias (a) and by $1.7 \mathrm{MeV}$ protons at $100 \mathrm{~V}$ reversed bias (b) 